\title{
Study of the Characteristics of Dust Acoustic Solitary Waves and Dust Acoustic Shock Waves in Electron Free Dusty Space Plasma
}

\author{
Raicharan Denra, Samit Paul, Susmita Sarkar \\ Department of Applied Mathematics, University of Calcutta, Kolkata, India \\ Email: raicharanroykolkata@gmail.com, samitpaul4@gmail.com, susmita62@yahoo.co.in
}

How to cite this paper: Denra, R., Paul, S. and Sarkar, S. (2018) Study of the Characteristics of Dust Acoustic Solitary Waves and Dust Acoustic Shock Waves in Electron Free Dusty Space Plasma. Journal of Modern Physics, 9, 948-960.

https://doi.org/10.4236/jmp.2018.95058

Received: November 18, 2017

Accepted: April 9, 2018

Published: April 12, 2018

Copyright (c) 2018 by authors and Scientific Research Publishing Inc. This work is licensed under the Creative Commons Attribution International License (CC BY 4.0).

http://creativecommons.org/licenses/by/4.0/

\begin{abstract}
A theoretical investigation has been done for the study of dust acoustic solitary waves and dust acoustic shock waves propagating in an unmagnetized, collisionless Lorentzian dusty plasma considering adiabatic and non-adiabatic dust charge variation. Plasma under consideration is composed of inertialess Lorentzian positive and negative ions along with inertial positively charged dust grains. Such dust grains are charged by the flow of positive ion and negative ion current over the grain surface. Adiabatic grain charge variation shows the existence of compressive soliton whose amplitude decreases and width increases with increasing number of suprathermal particles. Non-adiabatic dust charge variation is concerned with the propagation of monotonic dust acoustic shock waves which do not loose monotonicity even when a number of suprathermal particles are very large.
\end{abstract}

\section{Keywords}

Dust Acoustic Soliton, Dust Acoustic Shock, Kappa Distribution, Dusty Space Plasma, Positive Dust Charge

\section{Introduction}

An almost electron free plasma state is realized in negative ion containing plasmas which often appears in plasma processing such as deposition and etching etc., and also in the D-layer of the earth's ionosphere. Behavior of such electron free positive ion-negative ion plasma differs from usual electron-ion plasma as the mass ratio in this case does not differ much like electron-ion plasma. Such plasma of heavy charged particles can make an ion source of bipolar type and can be extended to dusty plasmas. A Japanese rocket [1] at a winter pre-dawn 
ionosphere detected a negative ion rich state (negative ion to positive ion ratio $0.9)$ at $75-80 \mathrm{~km}$ which decreases above $86 \mathrm{Km}$ toward the E layer [2]. Amemiya and Nakamura [3] in Japan performed another experiment using a Faraday cup showing the extension of negative-ion layer from about $60 \mathrm{~km}$ toward the height near $90 \mathrm{~km}$. Thus D-layer of the earth's ionosphere which is extended from $50 \mathrm{~km}-90 \mathrm{~km}$ above earth's surface sustains a negative ion-rich plasma which is almost electron free.

Negative ions in plasma are formed by different mechanisms like electron attachment, dissociative attachment, charge transfer and clustering reactions. They are frequently observed in afterglow when source of ionization is removed. Thus lower part of the ionosphere is a rich source of negative ions where solar radiation does not reach at night. In such plasmas electrons can be completely escaped after finite time and ion-ion plasma is produced [4] [5].

This D-layer of the ionosphere may contain dust or dust grains may be artificially injected there from outside. Plasma irregularities associated with the creation of an artificial dust layer in the Earth's ionosphere were investigated with a 2-D plasma simulation model. Electron irregularities were observed due to growth of a plasma instability driven by inhomogeneities in the boundary between the background plasma and the expanding charged dust layer [6]. Formation of nanoparticles or droplets during melting and vaporization of solids may give rise to many new interesting phenomena like ball lightning and anomalous conductivity [7] [8].

Effect of negative ions on charging of dust grains was investigated by Kim and Merlino in laboratory experiment [9]. They produced dusty plasma with positive grain charge in a $\mathrm{Q}$-machine $\mathrm{K}^{+}$plasma by the addition of $\mathrm{SF}_{6}$ gas. Negative ions $\left(\mathrm{SF}_{6}^{-}\right)$replace the plasma electrons, and the dust grains acquire a positive charge, since the $\mathrm{K}^{+}$ions are more mobile than the $\mathrm{SF}_{6}$ ions. They analyzed the excitations of ion-acoustic and dust acoustic waves by an electric field. The same mechanism of dust charging is also applicable in electron free space plasmas.

Space and astrophysical plasmas consist of suprathermal charge particles whose existence was proved by space craft observation [9] [10]. Charge particle in such plasmas follow the generalized kappa velocity distribution [11] and is known as Lorentzian plasmas. In ionosphere radio wave interact with charge particles. These charge particles in resonance condition accelerate with high velocity. Such high velocity particles are suprathermal in nature and follow generalized kappa velocity distribution. Suprathermal positive and negative ions can be achieved in the $\mathrm{D}$ layer of the ionosphere when radio waves in the low frequency range interact with positive and negative ions. Such suprathermal positive and negative ions along with dust grains form a Lorentzian dusty plasma where the dust grains become positively charged by the above mentioned mechanism.

Presence of negative ions produces many nonlinear phenomena in space plasmas e.g., nonlinear plasma decay in the afterglow, ion density peaks near discharge periphery, complex sheath structure etc. Nonlinear low frequency 
wave propagation in electronegative dusty plasma with adiabatic dust charge variation in presence of magnetic field with Maxwellian charge particles was earlier reported [12]. Shahmansouri and other scientist have studied dust acoustic solitary waves in electron depleted superthermal dusty plasmas considering the influence of dust charge variation [13] [14] [15] [16]. In this paper we shall investigate the characteristics of nonlinear wave propagation in an electron free Lorentzian dusty plasma with both adiabatic and non adiabatic dust charge variation.

\section{Mathematical Formulation}

To study the non linear propagation of dust acoustic waves in electron free dusty plasma we have considered positively charged cold, inertial dust grains and Kappa distributed inertia less positive and negative ions. Both adiabatic and non adiabatic fluctuation of grain charge has been considered. Since frequency of the dust acoustic wave is very low, its phase velocity is very small compared to positive and negative ion thermal velocities. Consequently positive and negative ions will not respond much to such very low frequency wave. Hence their inertia can be neglected, only dust inertia in this case is important. OML theory [17] has been used to calculate the positive ion and negative current flowing to the dust grains. In the plasma medium under our consideration equilibrium charge neutrality condition reads as

$$
n_{i 0}+z_{d 0} n_{d 0}=n_{n 0}
$$

where $n_{i 0}, n_{n 0}, n_{d 0}$ are respectively the positive ion, negative ion and dust number densities in equilibrium, and $z_{d 0}$ is the unperturbed number of charges residing on the dust grain measured in the unit of electron charge. For one-dimensional low-frequency dust acoustic wave motion the number density $n_{d}$ and average velocity $u_{d}$ of the cold dust fluid satisfies the particle and momentum conservation equations

$$
\begin{gathered}
\frac{\partial n_{d}}{\partial t}+\frac{\partial}{\partial x}\left(n_{d} u_{d}\right)=0 \\
\frac{\partial u_{d}}{\partial t}+u_{d} \frac{\partial u_{d}}{\partial x}=-\frac{q_{d}}{m_{d}} \frac{\partial \phi}{\partial x}
\end{gathered}
$$

along with the Poisson equation

$$
\frac{\partial^{2} \phi}{\partial x^{2}}=-4 \pi\left(q_{i} n_{i}-q_{n} n_{n}+q_{d} n_{d}\right)
$$

Number densities $n_{n}$ and $n_{i}$ of inertia less Kappa distributed negative and positive ions are,

$$
n_{n}=n_{n 0}\left(1-\frac{2 e \phi}{m_{n} k_{n} \theta_{n}^{2}}\right)^{-\left(k_{n}-\frac{1}{2}\right)} \text { and } n_{i}=n_{i 0}\left(1+\frac{2 e \phi}{m_{i} k_{i} \theta_{i}^{2}}\right)^{-\left(k_{i}-\frac{1}{2}\right)}
$$

Here $\kappa_{n}$ and $\kappa_{i}$ are negative ion and positive ion Kappa indices, $m_{d}, m_{i}$ and $m_{n}$ are the dust, positive ion and negative ion masses and $\varphi$ is the back 
ground plasma potential. $\theta_{n}=\sqrt{\frac{\left(k_{n}-\frac{3}{2}\right)}{k_{n}}\left(\frac{2 T_{n}}{m_{n}}\right)}$ and $\theta_{i}=\sqrt{\frac{\left(k_{i}-\frac{3}{2}\right)}{k_{i}}\left(\frac{2 T_{i}}{m_{i}}\right)}$ with negative and positive ion temperatures $T_{n}, T_{i}$ are thermal velocities of suprathermal negative ions and positive ions respectively. $q_{i}, q_{n}, q_{d}$ are the charge of positive ion, negative ion and dust grain respectively. The variable dust charge $q_{d}\left(=z_{d} e\right)$ in Equation (3) satisfies the grain charging equation

$$
\frac{\mathrm{d} q_{d}}{\mathrm{~d} t}=I_{n}+I_{i} .
$$

where

$$
\begin{aligned}
& I_{n}=-\pi r_{0}^{2} e n_{n}\left(\frac{8 T_{n}}{\pi m_{n}}\right)^{\frac{1}{2}}\left(k_{n}-\frac{3}{2}\right)^{\frac{1}{2}} \frac{\Gamma\left(k_{n}-1\right)}{\Gamma\left(k_{n}-\frac{1}{2}\right)}\left(1+\frac{e q_{d}\left(k_{n}-1\right)}{r_{0}\left(k_{n}-\frac{3}{2}\right) T_{n}}\right) \\
& I_{i}=\pi r_{0}^{2} e n_{i}\left(\frac{8 T_{i}}{\pi m_{i}}\right)^{\frac{1}{2}}\left(k_{i}-\frac{3}{2}\right)^{\frac{1}{2}} \frac{\Gamma\left(k_{i}-1\right)}{\Gamma\left(k_{i}-\frac{1}{2}\right)}\left(1+\frac{e q_{d}}{r_{0}\left(k_{i}-\frac{3}{2}\right) T_{i}}\right)^{-\left(k_{i}-1\right)}
\end{aligned}
$$

are the negative and positive ion currents flowing to the dust grains, calculated using OML theory [15] and $r_{0}$ is the radius of the dust grain.

Above equations have been non-dimensionalized in the following way. Negative ion, positive ion and dust number densities $n_{n}, n_{i}, n_{d}$ are normalized by corresponding unperturbed number densities $n_{n o}, n_{i o}$ and $n_{d o}$. The dust charge number $z_{d}$ is normalized by its equilibrium value $z_{d 0}$. The space coordinates $x$, time $t$, grain charge $q_{d}$, electrostatic potential $\phi$ and average dust velocity $u_{d}$ are respectively normalized by the Debye length

$\lambda_{D d}=\left(\frac{T_{e f f}}{4 \pi z_{d 0} n_{d 0} e^{2}}\right)^{\frac{1}{2}}$, the inverse of dust plasma frequency $\omega_{p d}^{-1}=\left(\frac{m_{d}}{4 \pi n_{d 0} z_{d 0}^{2} e^{2}}\right)^{\frac{1}{2}}$, equilibrium grain charge $e z_{d 0}$, the negative ion thermal energy $T_{n}$ (in $\mathrm{eV}$ ) and the dust-acoustic speed $c_{d}=\left(\frac{z_{d 0} T_{\text {eff }}}{m_{d}}\right)^{\frac{1}{2}}$. Here

$$
T_{\text {eff }}=T_{n} \alpha_{d}, \alpha_{d}=\frac{(1-\delta)}{\left\{\frac{\left(\kappa_{n}-\frac{1}{2}\right)}{\left(\kappa_{n}-\frac{3}{2}\right)}+\frac{\delta\left(\kappa_{i}-\frac{1}{2}\right)}{\sigma\left(\kappa_{i}-\frac{3}{2}\right)}\right\}}
$$

is the effective dusty plasma temperature calculated from the relation

$$
\frac{1}{T_{\text {eff }}}=\frac{1}{Z_{\mathrm{do}} n_{\mathrm{do}}}\left(\frac{\left(\kappa_{n}-\frac{1}{2}\right)}{\left(\kappa_{n}-\frac{3}{2}\right)} \frac{n_{n o}}{T_{n}}+\frac{\left(\kappa_{i}-\frac{1}{2}\right)}{\left(\kappa_{i}-\frac{3}{2}\right)} \frac{n_{i 0}}{T_{i}}\right) \text {, where } \delta=\frac{n_{i 0}}{n_{n 0}}, \sigma=\frac{T_{i}}{T_{n}} \text {. Therefore, }
$$


we obtain a set of normalized basic equations

$$
\begin{gathered}
\frac{\partial N_{d}}{\partial T}+\frac{\partial}{\partial x}\left(N_{d} V_{d}\right)=0 \\
\frac{\partial V_{d}}{\partial T}+V_{d} \frac{\partial V_{d}}{\partial X}=-\frac{Q}{\alpha_{d}} \frac{\partial \Phi}{\partial X} \\
\frac{\partial^{2} \Phi}{\partial X^{2}}=-\frac{\alpha_{d}}{(1-\delta)}\left\{(1-\delta) Q N_{d}-N_{n}+\delta N_{i}\right\} \\
\left(\frac{\omega_{p d}}{v_{d}}\right)\left(\frac{\partial Q}{\partial T}+V_{d} \frac{\partial Q}{\partial X}\right)=\frac{1}{v_{d} e Z_{d 0}}\left(I_{n}+I_{i}\right) .
\end{gathered}
$$

with the normalized negative and positive ion number densities

$$
N_{n}=\left\{1-\frac{\Phi}{\kappa_{n}-\frac{3}{2}}\right\}^{-\left(\kappa_{n}-\frac{1}{2}\right)} \quad, N_{i}=\left\{1+\frac{\Phi}{\sigma\left(\kappa_{i}-\frac{3}{2}\right)}\right\}^{-\left(\kappa_{i}-\frac{1}{2}\right)}
$$

and the current expressions

$$
\begin{aligned}
& I_{n}=-\pi r_{0}^{2} e \sqrt{\frac{8 T_{n}}{\pi m_{n}}} \frac{\left(\kappa_{n}-\frac{3}{2}\right)^{\frac{1}{2}} \Gamma\left(\kappa_{n}-1\right)}{\Gamma\left(\kappa_{n}-\frac{1}{2}\right)} n_{n 0} N_{n}\left\{1+\frac{Z Q\left(\kappa_{n}-1\right)}{\left(\kappa_{n}-\frac{3}{2}\right)}\right\} \\
& I_{i}=\pi r_{0}^{2} e \sqrt{\frac{8 T_{i}}{\pi m_{i}}} \frac{\left(\kappa_{i}-\frac{3}{2}\right)^{\frac{1}{2}} \Gamma\left(\kappa_{i}-1\right)}{\Gamma\left(\kappa_{i}-\frac{1}{2}\right)} n_{i 0} N_{i}\left\{1+\frac{Z Q}{\sigma} \frac{1}{\left(\kappa_{i}-\frac{3}{2}\right)}\right\}^{-\left(\kappa_{i}-1\right)}
\end{aligned}
$$

where normalization has been done in the following form.

$$
\begin{aligned}
& Z=\frac{e^{2} z_{d 0}}{r_{0} T_{n}}, Q=\frac{q_{d}}{e z_{d 0}}, X=\frac{X}{\lambda_{d}}, T=\frac{t}{\omega_{p d}^{-1}}, V=\frac{u_{d}}{c_{d}}, \\
& \Phi=\frac{e \varphi}{T_{n}}, N_{d}=\frac{n_{d}}{n_{d 0}}, N_{n}=\frac{n_{n}}{n_{n 0}}, N_{i}=\frac{n_{i}}{n_{i 0}}, \sigma=\frac{T_{i}}{T_{n}}
\end{aligned}
$$

The grain charging frequency $v_{d}$ has been calculated in the form,

$$
\begin{aligned}
v_{d}= & \frac{r_{0}}{\sqrt{2 \pi}} \frac{\omega_{p i}}{V_{t h i}} \frac{\Gamma\left(\kappa_{i}\right)}{\left(\kappa_{i}-\frac{3}{2}\right)^{\frac{1}{2}} \Gamma\left(\kappa_{i}-\frac{1}{2}\right)} \\
& \left.\times\left\{1+\frac{Z}{\sigma\left(\kappa_{i}-\frac{3}{2}\right)}\right\}^{-\kappa_{i}}+\sigma \frac{\left(\kappa_{i}-\frac{3}{2}\right)\left(\kappa_{n}-1\right)}{\left(\kappa_{i}-1\right)\left(\kappa_{n}-\frac{3}{2}\right)} \frac{\left(\kappa_{i}-\frac{3}{2}\right) \sigma}{\left\{1+\frac{Z\left(\kappa_{n}-1\right)}{\left(\kappa_{n}-1\right)}\right\}}\right\}
\end{aligned}
$$


Here $r_{0}$ is the grain radius, $\omega_{p i}$ and $V_{t h i}$ are the ion plasma frequency and the ion thermal velocity respectively. In the following two subsections we shall investigate the two cases of adiabatic and non adiabatic dust charge variation.

In equilibrium substitution of the expressions of $I_{n}$ and $I_{i}$ from Equation (15) and (16) in the current balance equation provides the positive ion-negative ion density ratio $\delta\left(=\frac{n_{i 0}}{n_{n 0}}\right)$ in the form,

$$
\begin{aligned}
\delta= & A\left\{1-\frac{\Phi}{\kappa_{n}-\frac{3}{2}}\right\}^{-\left(\kappa_{n}-\frac{1}{2}\right)}\left\{1+\frac{Z Q\left(\kappa_{n}-1\right)}{\left(\kappa_{n}-\frac{3}{2}\right)}\right\} /\left\{1+\frac{\Phi}{\sigma\left(\kappa_{i}-\frac{3}{2}\right)}\right\}^{-\left(\kappa_{i}-\frac{1}{2}\right)} \\
& \times\left\{1+\frac{Z Q}{\sigma} \frac{1}{\left(\kappa_{i}-\frac{3}{2}\right)}\right\}^{-\left(\kappa_{i}-1\right)}
\end{aligned}
$$

In equilibrium ( $\Phi=0, Q=+1)(18)$ gives,

$$
\delta=\frac{n_{i 0}}{n_{n 0}}=A\left\{1+\frac{Z\left(\kappa_{n}-1\right)}{\left(\kappa_{n}-\frac{3}{2}\right)}\right\} /\left\{1+\frac{Z}{\sigma} \frac{1}{\left(\kappa_{i}-\frac{3}{2}\right)}\right\}^{-\left(\kappa_{i}-1\right)}
$$

where $A=\frac{\left(\kappa_{n}-\frac{3}{2}\right)^{\frac{1}{2}} \Gamma\left(\kappa_{n}-1\right) \Gamma\left(\kappa_{i}-\frac{1}{2}\right)}{\left(\kappa_{i}-\frac{3}{2}\right)^{\frac{1}{2}} \Gamma\left(\kappa_{i}-1\right) \Gamma\left(\kappa_{n}-\frac{1}{2}\right)} \sqrt{\frac{m_{i}}{\sigma m_{n}}}$.

For the study of small-amplitude nonlinear dust acoustic waves in the presence of self-consistent adiabatic dust-charge variation, we employ reductive perturbation technique to the Equations (10)-(14) using stretched coordinates $\xi=\varepsilon^{\frac{1}{2}}(X-\lambda T)$, and $\tau=\varepsilon^{\frac{3}{2}} T$, where $\varepsilon$ is a small parameter and $\lambda$ is unknown normalized phase velocity of the linear dust acoustic waves.

The variables $N_{d}, V_{d}, \Phi$, and $Q$ are then expanded as

$$
\begin{gathered}
N_{d}=1+\varepsilon N_{d 1}+\varepsilon^{2} N_{d 2}+\varepsilon^{3} N_{d 3}+\cdots, \\
V_{d}=\varepsilon V_{d 1}+\varepsilon^{2} V_{d 2}+\varepsilon^{3} V_{d 3}+\cdots \\
\Phi=\varepsilon \Phi_{1}+\varepsilon^{2} \Phi_{2}+\varepsilon^{3} \Phi_{3}+\cdots, \\
Q=1+\varepsilon Q_{1}+\varepsilon^{2} Q_{2}+\varepsilon^{3} Q_{3}+\cdots
\end{gathered}
$$

In this case expansion of $Q$ has started from +1 as equilibrium dust charge is positive.

Substituting these expansions into Equations (10)-(14) and (18) and comparing coefficient of $\varepsilon$ we have, 


$$
\begin{gathered}
N_{d 1}=\frac{\Phi_{1}}{\alpha_{d} \lambda^{2}} \\
Q_{1}=\frac{1}{\alpha_{d}}\left(1-\frac{1}{\lambda^{2}}\right) \Phi
\end{gathered}
$$

To the next higher order in $\varepsilon$ we have the following set of equations

$$
\begin{gathered}
\frac{\partial N_{d 1}}{\partial \tau}-\lambda \frac{\partial N_{d 2}}{\partial \xi}+\frac{\partial V_{d 2}}{\partial \xi}+\frac{\partial\left(N_{d 1} V_{d 1}\right)}{\partial \xi}=0 \\
\frac{\partial V_{d 1}}{\partial \tau}-\lambda \frac{\partial V_{d 2}}{\partial \xi}+V_{d 1} \frac{\partial V_{d 1}}{\partial \xi}=-\frac{1}{\alpha_{d}}\left(\frac{\partial \Phi_{2}}{\partial \xi}+Q_{1} \frac{\partial \Phi_{1}}{\partial \xi}\right) \\
\frac{\partial^{2} \Phi_{1}}{\partial \xi^{2}}=-\alpha_{d} N_{d 2}-\alpha_{d} Q_{2}+\Phi_{2}-E \Phi_{1}^{2}
\end{gathered}
$$

where $E=\frac{1}{\lambda^{2} \alpha_{d}}\left(1-\frac{1}{\lambda^{2}}\right)+\frac{1}{2}\left\{\frac{\frac{\left(\kappa_{i}-\frac{1}{2}\right)\left(\kappa_{i}+\frac{1}{2}\right)}{\left(\kappa_{i}-\frac{3}{2}\right)^{2}} \frac{\delta}{\sigma^{2}}-1}{1+\frac{\delta}{\sigma} \frac{\left(\kappa_{i}-\frac{1}{2}\right)}{\left(\kappa_{i}-\frac{3}{2}\right)}}\right\}$.

The above set of equations is common to both adiabatic and nonadiabatic dust charge variation. But the reductive perturbation in the grain charging Equation (13) will be different in these two different cases. We shall now consider the Equation (13) separately for adiabatic and non-adiabatic dust charge variation.

\subsection{Adiabatic Dust Charge Variation}

For adiabatic dust charge variation, dust charging time is very small and hence dust charging frequency is very high compared to dust plasma frequency which implies $\frac{\omega_{p d}}{v_{d}} \approx 0$, so (13) gives the current balance equation $I_{n}+I_{i}=0$.

Equating from both sides the terms containing $\varepsilon$ and $\varepsilon^{2}$, we get $Q_{d 1}$ and $Q_{d 2}$ in the following form

where $\beta_{d}=\frac{\left\{\frac{\left(\kappa_{n}-\frac{1}{2}\right)}{\left(\kappa_{n}-\frac{3}{2}\right)}\left(1+Z \frac{\left(\kappa_{n}-1\right)}{\left(\kappa_{n}-\frac{3}{2}\right)}\right) \frac{A}{\delta}+B \frac{\left(\kappa_{i}-\frac{1}{2}\right)}{\left(\kappa_{i}-\frac{3}{2}\right)} \frac{1}{\sigma}\right\}}{\left\{\frac{A Z}{\delta} \frac{\left(\kappa_{n}-1\right)}{\left(\kappa_{n}-\frac{3}{2}\right)}-\frac{\left(\kappa_{i}-1\right)}{\left(\kappa_{i}-\frac{3}{2}\right)} \frac{Z}{\sigma}\left(\frac{\kappa_{i} Z}{\left(\kappa_{i}-\frac{3}{2}\right)}-1\right)\right\}}$ 


$$
\begin{aligned}
B=1-\frac{\left(\kappa_{i}-1\right)}{\left(\kappa_{i}-\frac{3}{2}\right)} \frac{Z}{\sigma}+\frac{\left(\kappa_{i}-1\right) \kappa_{i}}{2\left(\kappa_{i}-\frac{3}{2}\right)^{2} \frac{Z^{2}}{\sigma^{2}}} & r_{d}=\frac{r_{d 1}}{r_{d 2}}, r_{d 2}=\frac{\left(\kappa_{n}-1\right)}{\left(\kappa_{n}-\frac{3}{2}\right)} \frac{A Z}{\delta}-\frac{Z\left(\kappa_{i}-1\right)}{\sigma\left(\kappa_{i}-\frac{3}{2}\right)}\left\{\frac{\kappa_{i}}{\left(\kappa_{i}-\frac{3}{2}\right)} \frac{Z}{\sigma}-1\right\} \text { and } \\
r_{d 1}= & A \frac{Z \beta_{d}}{\delta} \frac{\left(\kappa_{n}-\frac{1}{2}\right)\left(\kappa_{n}-1\right)}{\left(\kappa_{n}-\frac{3}{2}\right)^{2}}+\frac{\left(\kappa_{i}-1\right) \kappa_{i}}{\left(\kappa_{i}-\frac{3}{2}\right)^{2}} \frac{Z^{2} \beta_{d}^{2}}{\sigma^{2}} \\
- & \frac{\left(\kappa_{n}-\frac{1}{2}\right)\left(\kappa_{n}+\frac{1}{2}\right)}{2\left(\kappa_{n}-\frac{3}{2}\right)^{2}}\left\{1+\frac{\left(\kappa_{n}-1\right)}{\left(\kappa_{n}-\frac{3}{2}\right)} Z\right\} \frac{A}{\delta}+\frac{\left(\kappa_{i}-\frac{1}{2}\right)\left(\kappa_{i}+\frac{1}{2}\right)}{2\left(\kappa_{i}-\frac{3}{2}\right)^{2}} \frac{B}{\sigma^{2}} \\
& -\frac{\left(\kappa_{i}-1\right)\left(\kappa_{i}-\frac{1}{2}\right)}{\left(\kappa_{i}-\frac{3}{2}\right)^{2}} \frac{Z \beta_{d}}{\sigma^{2}}\left\{\frac{\kappa_{i}}{\left(\kappa_{i}-\frac{3}{2}\right)} \frac{Z}{\sigma}-1\right\}
\end{aligned}
$$

To get an evolution equation of $\Phi_{1}$ we have eliminated all second order terms from Equations (21)-(24) and obtained the KdV equation,

$$
\begin{gathered}
\frac{\partial \Phi_{1}}{\partial \tau}+a \Phi_{1} \frac{\partial \Phi_{1}}{\partial \xi}+b \frac{\partial^{3} \Phi_{1}}{\partial \xi^{3}}=0 . \\
\text { where } b=\frac{1}{2\left(1+\alpha_{d} \beta_{d}\right)^{\frac{3}{2}}}, a=\alpha_{d} b\left[2 r_{d}+\frac{3}{\alpha_{d}^{2} \lambda^{4}}-\frac{\beta_{d}}{\alpha_{d} \lambda^{2}}+\frac{2 E}{\alpha_{d}}\right]
\end{gathered}
$$

The stationary solution of this $\mathrm{KdV}$ equation is $\phi_{1}=\phi_{1 m} \operatorname{sech}^{2}[(\xi-M \tau) / W]$ which represents soliton of amplitude $\phi_{1 m}=\frac{3 M}{a}$ and width $W=2 \sqrt{\frac{b}{M}}$.

Here $M$ is the Mach number which is the ratio of the wave velocity and the velocity of sound. Nature of this soliton whether compressive or rarefactive will be determined from numerical computation.

\subsection{Nonadiabatic Dust Charge Variation}

On the other hand nonadiabaticity of dust charge fluctuation provides an alternate physical mechanism causing dissipation. To study the effect of this nonadiabaticity we assume $\frac{\omega_{p d}}{v_{d}}=v \sqrt{\varepsilon}$ where $\varepsilon$ is small and $v$ is of the order of unity. Then the reductive perturbation technique gives from Equation (13) the first and second order dust charge perturbation in the form,

$$
Q_{1}=-\beta_{d} \Phi_{1} \text { and } Q_{2}=-\beta_{d} \Phi_{2}+r_{d} \Phi_{1}^{2}-\mu_{1} \frac{\partial \Phi_{1}}{\partial \xi}
$$




$$
\begin{aligned}
& \text { where } \mu_{1}=\frac{v \lambda \beta_{d}}{C\left\{\frac{A Z}{\delta} \frac{\left(\kappa_{n}-1\right)}{\left(\kappa_{n}-\frac{3}{2}\right)}-\frac{Z^{2}}{\sigma^{2}} \frac{\left(\kappa_{i}-1\right) \kappa_{i}}{\left(\kappa_{i}-\frac{3}{2}\right)^{2}}+\frac{Z}{\sigma} \frac{\left(\kappa_{i}-1\right)}{\left(\kappa_{i}-\frac{3}{2}\right)}\right\}} \text { and } \\
& C=\frac{\left(\kappa_{i}-\frac{3}{2}\right) \sigma}{\left[\left\{1+\frac{Z}{\sigma\left(\kappa_{i}-\frac{3}{2}\right)}\right\}^{-\kappa_{i}}+\sigma \frac{\left(\kappa_{i}-\frac{3}{2}\right)\left(\kappa_{n}-1\right)}{\left(\kappa_{i}-1\right)\left(\kappa_{n}-\frac{3}{2}\right)} \frac{\left\{1+\frac{Z}{\left(\kappa_{i}-\frac{3}{2}\right) \sigma}\right\}}{\left\{1+\frac{Z\left(\kappa_{n}-1\right)}{\left(\kappa_{n}-\frac{3}{2}\right)}\right\}}\right]}
\end{aligned}
$$

Eliminating all the $2^{\text {nd }}$ order terms from (21)-(24) and from (27) we get the $\mathrm{KdV}$-Burger equation,

$$
\frac{\partial \Phi_{1}}{\partial \tau}+a \Phi_{1} \frac{\partial \Phi_{1}}{\partial \xi}+b \frac{\partial^{3} \Phi_{1}}{\partial \xi^{3}}=\mu \frac{\partial^{2} \Phi_{1}}{\partial \xi^{2}}
$$

where $\mu=\frac{\mu_{1} \lambda^{3} \alpha_{d}}{2}$ is the Burger term representing the nonadiabaticity induced dissipation which was absent in the adiabatic case. This is a viscous like effect as in the case of hydrodynamic fluid flow. This viscous like dissipative effect may be generated due to the relative velocity between the positive and negative ion fluids and dust fluid layers and is coming into the picture for comparatively slow charging process in case of nonadiabatic dust charge variation.

\section{Numerical Estimation}

For numerical estimation of this problem we consider an electron free space plasma with suprathermal positive and negative ions and positively charged dust grains whose quasineutrality condition (1) implies that the equilibrium density ratio $\delta=\frac{n_{i 0}}{n_{n 0}}$ must be less than one. The temperature ratio $\sigma=\frac{T_{i}}{T_{n}}$ is greater than unity as negative ion mass is higher than positive ion mass. Equation (19) shows that $\delta$ is a function of $Z$. Using equation (19) we shall find the permissible range of the normalized grain charge number $z$ satisfying the condition $\delta<1$. Our main objective is to see the effect of suprathermal particles on amplitude and width of the dust acoustic soliton when dust charge variation is adiabatic and nature of the dust acoustic shock wave when dust charge variation is nonadiabatic. We have made the negative ion Maxwellian by doing $\kappa_{n} \rightarrow \infty$. All numerical calculations are depicted in figures where we fixed $\sigma=1.5, \quad M=1.1$ and $\kappa_{i}=3,4,6$. This approximation is valid as negative ion mass is large com- 
pared to positive ion mass.

Figure 1 shows the variation of $\delta$ with $Z$ for above mentioned set of kappa values. This figure indicates that permissible range of $Z$ is $0<Z<0.12$ satisfying $\delta<1$. We have ploted soliton amplitude in Figure 2 and soliton width in Figure 3 when dust charge fluctuation is adiabatic. These figures show that amplitude of compressive dust acoustic soliton decreases and width increases with decreasing $\kappa_{i}$, i.e. with increasing number of suprathermal positive ions (decreasing $\kappa_{i}$ ). This means higher population of suprathermal positive ions reduces the soliton velocity as the soliton velocity is directly proportional to the soliton amplitude and inversely proportional to the soliton width.

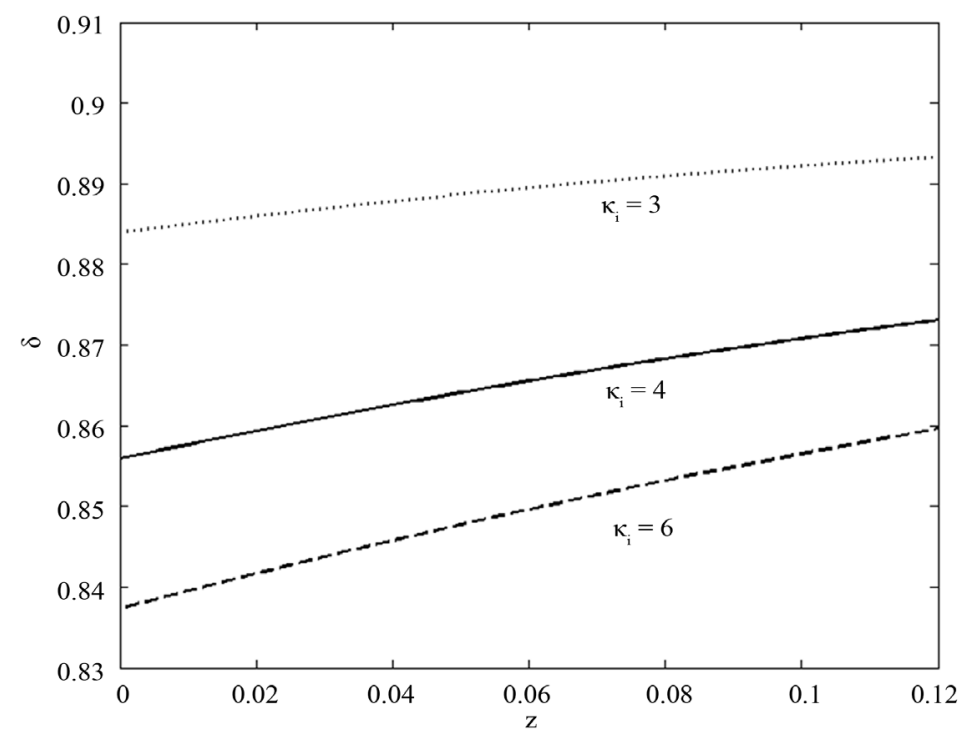

Figure 1. Plot of $\delta$ versus $z$ for different values of $\kappa_{i}$.

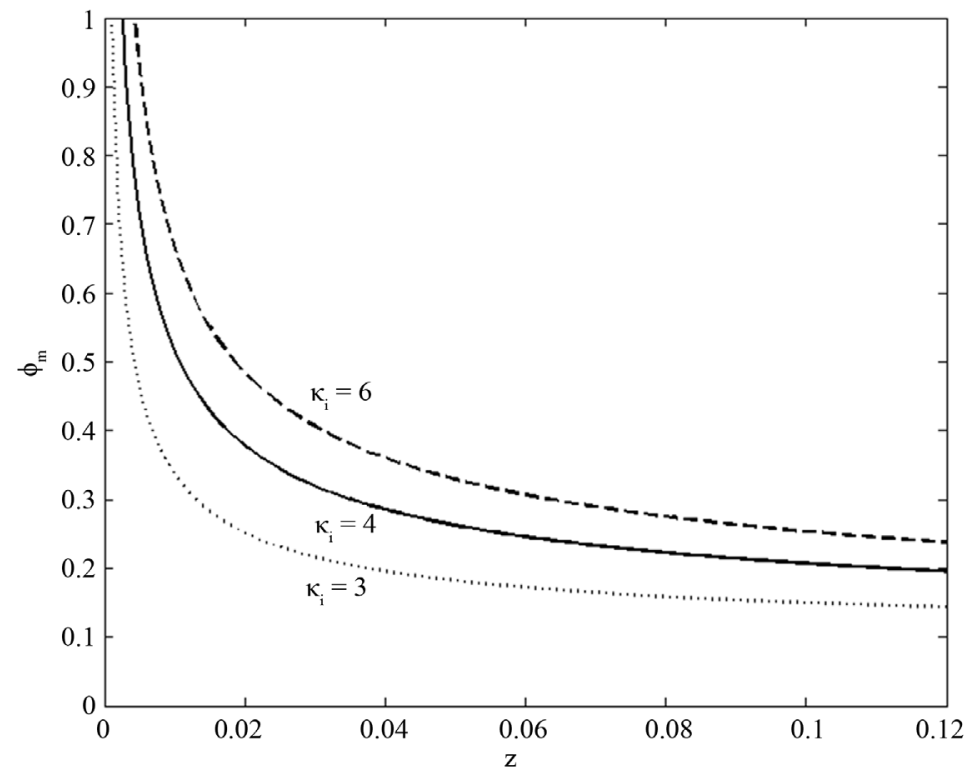

Figure 2. Plot of soliton amplitude $\phi_{m}$ versus $z$ for different values of $\kappa_{i}$. 
In case of nonadiabatic dust charge variation Figure 4 shows that the dissipation coefficient $\mu$ increases with decreasing $\kappa_{i}$. This implies increasing population of suprathermal positive ions ( $\kappa_{i}$ is low) increases nonadiabaticity induced dissipation $\mu$. Figure 5 shows that the dissipation-dispersion coefficient ratio $\mu / b$ does not reach below the value one for all values of $\kappa_{i}$ and hence in all cases dust acoustic shock wave is monotonic. Figure 5 also shows that montonicity is low for lower $\kappa_{i}\left(\kappa_{i}=3\right)$ i.e. for higher suprathermal positive ion population. Thus an electron free Lorentzian dusty space plasma composed of positive ion, negative ion and positively charged dust grains generates compressive

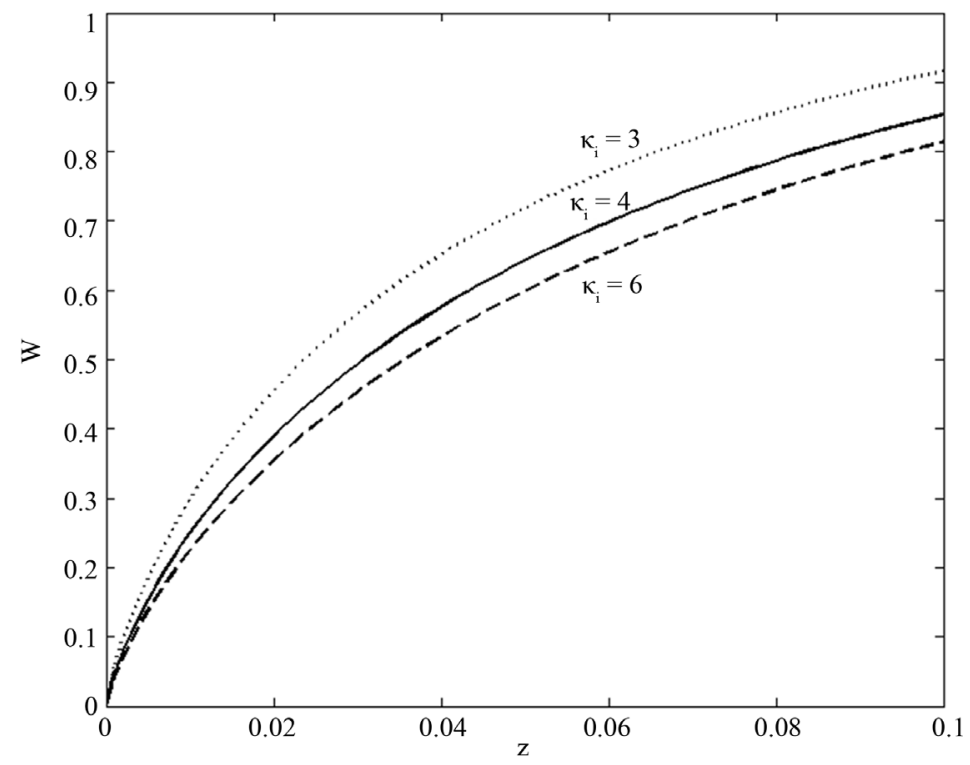

Figure 3. Plot of soliton width $W$ versus $z$ for different $\kappa_{i}$.

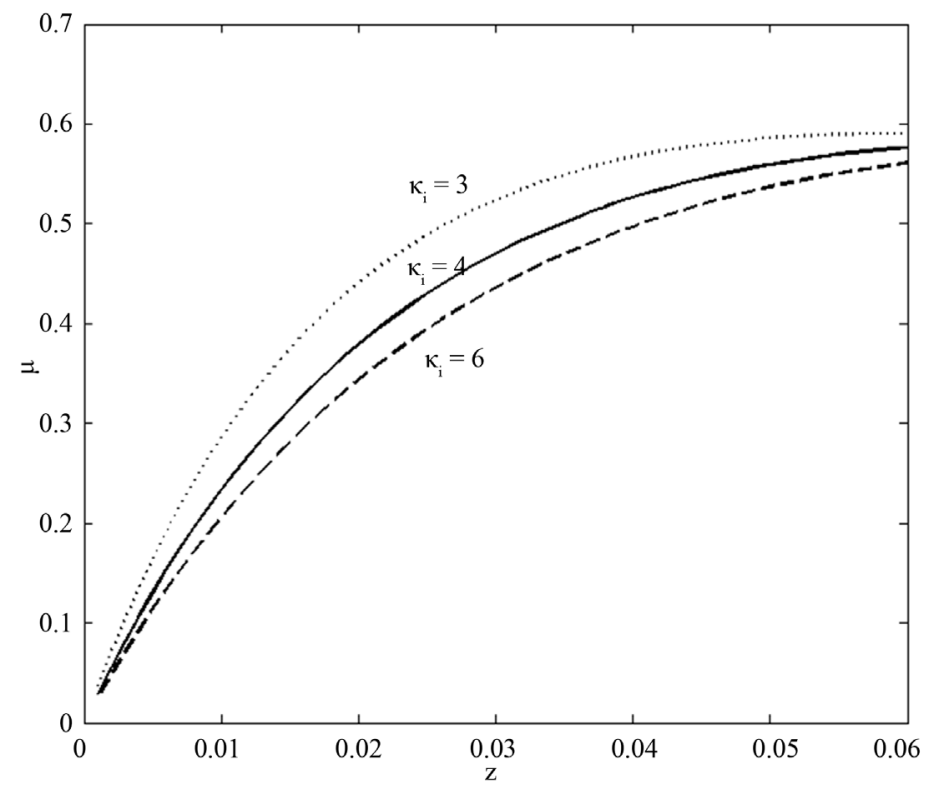

Figure 4. Plot of dissipation coefficient $\mu$ versus $z$ for different $\kappa_{i}$ 


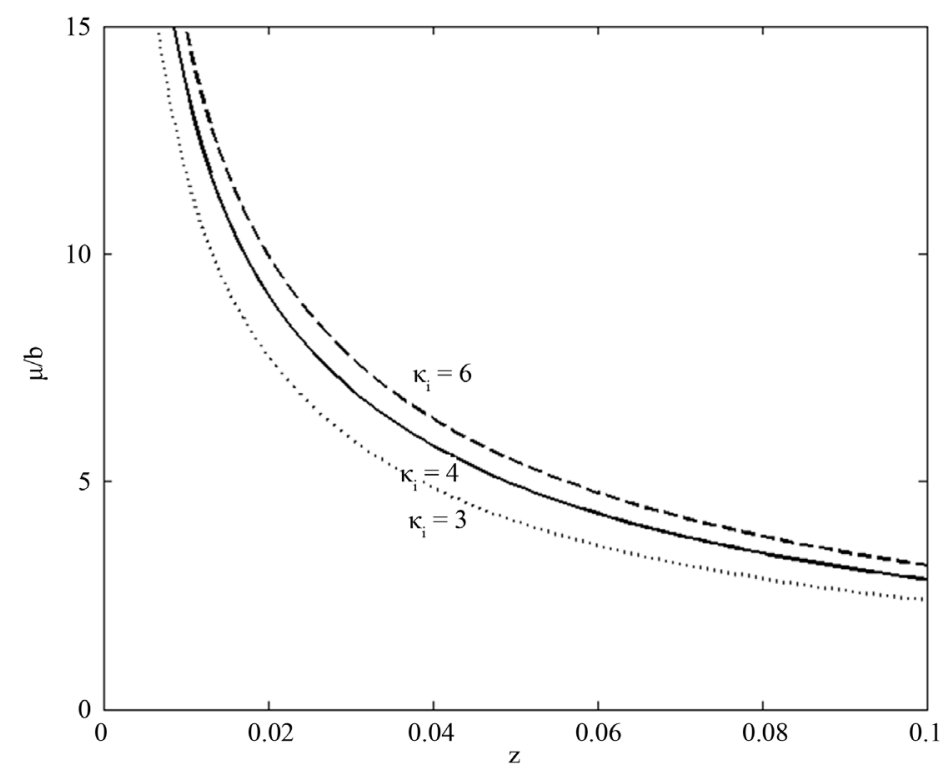

Figure 5. Plot of dissipation-dispersion ratio $\mu / b$ versus $z$ for different $\kappa_{i}$.

dust acoustic soliton for adiabatic dust charge variation whose amplitude decreases and width increases with increasing number of suprathermal positive ions. On the otherhand for non adiabatic dust charge variation such plasma generates monotonic dust acoustic shock wave whose monotonicity decreases with increasing suprathermal positive ion population.

\section{Conclusion}

In this paper we have studied nonlinear characteristics of small amplitude dust acoustic wave propagation in electron free Lorentzian dusty plasma considering positively charged cold, inertial dust grains and Kappa distributed inertia less positive and negative ions. In numerical estimation negative ions are made by Maxwellian of $\kappa_{n} \rightarrow \infty$. Dust grains are positively charged as heavy mass negative ion flux to the dust grains is less than positive ion flux. In this paper both adiabatic and nonadiabatic dust charge variations have been considered. Our analysis shows that in the permissible range of normalized grain charge number, for adiabatic dust charge variation compressive dust acoustic soliton propagates whose amplitude decreases and width increases with increasing number of suprathermal positive ions whereas for nonadiabatic dust charge variation monotonic dust acoustic shock wave propagates whose montonicity is lower for higher suprathermal positive ion population. In Reference [18] we studied nonlinear dust acoustic wave propagation in a Lorentzian dusty plasma with suprathermal electrons and ions and negatively charged dust grains where for adiabatic dust charge variation we obtained rarefied dust acoustic soliton with decreasing amplitude and increasing width with increasing suprathermal electron population. For nonadiabatic dust charge variation in that case we showed that dust acoustic shock wave was monotonic up to a certain range of grain charge number then it was oscillatory. 


\section{References}

[1] Takagi, M., Kondo, Y. and Iwata, A. (1980) Journal of Geomagnetism and Geoelectricity, 32, 715-719. https://doi.org/10.5636/jgg.32.715

[2] Amemiya, H. (1994) Dusty and Dirty Plasmas, Noise and Chaos in Space and the Laboratory. Kikuchi, H., Ed., Plenum Press, New York, 111.

[3] Amemiya, H. and Nakamura, Y. (1996) Journal of Geomagnetism and Geoelectricity, 48, 391-401. https://doi.org/10.5636/jgg.48.391

[4] Geortz, C.K. (1989) Reviews of Geophysics, 27, 271-292. https://doi.org/10.1029/RG027i002p00271

[5] Rapp, M., Hedin, J., Strelnikova, I., Friedrich, M., Gumbel, J. and Lubken, F.J. (2005) Geophysical Research Letters, 32, L23821. https://doi.org/10.1029/2005GL024676

[6] Scales, W., Bordikar, M.R., Mahmoudian, A. and Fu, H. (2010) IEEE Transactions on Plasma Science, 38, 880-885. https://doi.org/10.1109/TPS.2010.2041072

[7] Amemiya, H., Annaratone, B. and Allen, J. (1998) Journal of Plasma Physics, 60, 81-93. https://doi.org/10.1017/S0022377898006837

[8] Amemiya, H., Annaratone, B.M. and Allen, J.E. (1999) Plasma Sources Science and Technology, 8, 179. https://doi.org/10.1088/0963-0252/8/1/020

[9] Kim, S.-H. and Merlino, R.L. (2006) Physics of Plasmas, 13, Article ID: 052118.

[10] Northrop, T.G. (1992) Physica Scripta, 45, 475-490. https://doi.org/10.1088/0031-8949/45/5/011

[11] Pierrard, V. and Lazar, M. (2010) Solar Physics, 267, 153-174. https://doi.org/10.1007/s11207-010-9640-2

[12] Sarkar, S., Ghosh, S., Khan, M. and Gupta, M.R. (2011) Physics of Plasmas, 18, Article ID: 093703.

[13] Shahmansouri, M. and Tribeche, M. (2012) Astrophysics and Space Science, 342, 87-92. https://doi.org/10.1007/s10509-012-1149-8

[14] Shahmansouri, M. (2013) Iranian Journal of Science and Technology, 37, 285-291.

[15] Shahmansouri, M. and Alinejad, H. (2013) Astrophysics and Space Science, 343, 257-263. https://doi.org/10.1007/s10509-012-1216-1

[16] Shahmansouri, M. and Tribeche, M. (2013) Astrophysics and Space Science, 343, 257-263. https://doi.org/10.1007/s10509-012-1216-1

[17] Meyer-Vernet, N. (1982) Astronomy \& Astrophysics, 105, 98-106.

[18] Denra, R., Paul, S. and Sarkar, S. (2016) AIP Advances, 6, 125045. https://doi.org/10.1063/1.4972520 